Open Access

\title{
Toremifene, a selective estrogen receptor modulator, significantly improved biochemical recurrence in bone metastatic prostate cancer: a randomized controlled phase II a trial
}

Tetsuya Fujimura ${ }^{1 *}$, Satoru Takahashi ${ }^{2}$, Haruki Kume ${ }^{1}$, Tomohiko Urano ${ }^{3,4}$, Kenichi Takayama $^{3,4}$, Yuta Yamada$^{1}$, Motofumi Suzuki', Hiroshi Fukuhara', Tohru Nakagawa', Satoshi Inoue ${ }^{3,4}$ and Yukio Homma'

\begin{abstract}
Backgrounds: Durability of androgen-deprivation therapy (ADT) for prostate cancer (PC) is limited. Additional selective estrogen receptor modulators (SERMs) may prolong the durability of ADT, because androgen and estrogen signaling drive PC progression.

Methods: Men with treatment-naive bone metastatic PC were randomly assigned in 1:1:1 fashion to receive ADT, toremifene $60 \mathrm{mg}$ plus ADT (TOPADT), or raloxifene $60 \mathrm{mg}$ plus ADT (RAPADT). The primary endpoint was the biochemical recurrence $(B C R)$ rate, and secondary endpoints were changes of scores of the visual analogue scale (VAS) and the functional assessment of cancer therapy (FACT).
\end{abstract}

Results: A total of 15 men, 5 each, were allocated to one of the three treatment arms. The basal serum prostatespecific antigen (PSA) level was $198 \mathrm{ng} / \mathrm{mL}$ (median, range; 30-8428). Bone metastases were graded as $1(n=11), 2$ $(n=3)$, and $3(n=1)$ by the extent of disease. During the median follow-up period of 1370 days (range; 431-1983), BCR occurred in 3,0 and 2 men in ADT, TOPADT and RAPADT group, respectively. The 5-year BCR-free rate was 30, 100 and $53 \%$, in ADT, TOPADT and RAPADT group, respectively $(p=0.04$, ADT v.s. TOPADT, $p=0.48$, ADT v.s. RAPADT and $p=0.12$, TOPADT v.S. RAPADT). Scores of VAS improved in all groups and remained stable throughout the study. This analysis is limited as a preliminary result in a single center.

Conclusions: Toremifene with conventional ADT significantly improved the BCR rate in treatment-naive bone metastatic PC. Further clinical trials are warranted to confirm the promising clinical efficacy of this combination therapy.

Trial registration: The protocol was registered at the University Hospital Medical Information Network (UMIN ID;0,000,064,000) in Sep 25, 2011.

Keywords: Toremifene, Raloxifene, Androgen deprivation therapy, Biochemical recurrence, Prostate cancer

\footnotetext{
* Correspondence: tfujimura-jua@umin.ac.jp

'Department of Urology, Graduate School of Medicine, The University of

Tokyo, 7-3-1 Hongo, Bunkyo-ku, Tokyo 113-8655, Japan

Full list of author information is available at the end of the article
}

(c) 2015 Fujimura et al. Open Access This article is distributed under the terms of the Creative Commons Attribution 4.0 International License (http://creativecommons.org/licenses/by/4.0/), which permits unrestricted use, distribution, and reproduction in any medium, provided you give appropriate credit to the original author(s) and the source, provide a link to the Creative Commons license, and indicate if changes were made. The Creative Commons Public Domain Dedication waiver (http://creativecommons.org/publicdomain/zero/1.0/) applies to the data made available in this article, unless otherwise stated. 


\section{Background}

Based on the pioneering work by Huggins [1], androgen deprivation therapy (ADT) has been the primary treatment for advanced prostate cancer (PC). Unfortunately, most advanced cases of PC eventually become castrationresistant (CRPC), despite the continued use of ADT [2]. Novel therapies such as docetaxel, enzaltamide, abiraterone, cabazitaxel and sipuleucel-T [2-5] have been developed to treat CRPC. However, the development of agents that inhibit progression to CRPC may represent alternative therapeutic options for PC.

The results of recent studies have revealed growth regulation of PC via steroid nuclear receptors, which included not only the androgen receptor (AR) [6, 7] but also members of the estrogen receptor (ER) family $[8,9]$. $E R \alpha$ and $E R \beta c x(E R \beta 2)$ in particular have been implicated in PC progression and PC-related mortality, whereas ER $\beta$ inhibits tumor growth $[8,9]$. In this regard, selective estrogen receptor modulators (SERMs) are expected to change the clinical course of PC. For example, toremifene, an ER $\alpha$ antagonist in the prostate [10], decreased the incidence of PC in men with highgrade prostatic intraepithelial neoplasia (HGPIN) [11, 12]. Furthermoere, raloxifene inhibited androgen-independent PC growth in 5 (28\%) of 13 patients [13]. However, SERMs have not been fully investigated for use in those with treatment-naive PC. We hypothesized that additional SERMs may prolong the durability of ADT, because androgen and estrogen signaling drive $\mathrm{PC}$ progression. In the present study, we conducted a prospective randomized clinical phase IIA trial to investigate the effects of SERMs (toremifene and raloxifene) when added to ADT in treatment-naïve bone metastatic PC.

\section{Methods}

\section{Participants}

The inclusion criteria were men aged $\geq 20$ years if they had histological confirmed adenocarcinoma of the prostate and radiologically proven bone metastasis with performance status 0 , and adequate hepatic, hematological and renal function. Patients who had previous ADT or chemotherapy for PC, deep vein thrombosis, pulmonary embolism or antiphospholipid antibody syndrome were excluded. Bisphosphonate, warfarin, phenobarbital, rifampicin, phenitoin, ampicillin or cholestyramine was not allowed during the study.

Extent of diseases (EOD) of bone metastasis was graded by bone scintigraphy using technetium-99 m-methylene diphosphonate as follows: 0, normal or abnormal due to benign bone disease; 1 , number of bony metastases $<6$, each of which was $<50 \%$ of the size of a vertebral body (one lesion approximately the size of a vertebral body would be counted as two lesions); 2 , number of bone metastases between 6 and 20, size of lesions as previously described; 3 , number of metastases $\geq 20$ but less than a "super scan", and 4, "super scan" or its equivalent, i.e., more than $75 \%$ of the ribs, vertebrae and pelvic bones [14]. The Japan Cancer of the Prostate Risk Assessment (J-CAPRA) score (range; 0-12) was calculated on the basis of GS, PSA levels and clinical stage [15].

The protocol was approved by the ethical committee (Internal Review Board) at the University of Tokyo Hospital in August 2008 (approval number; P2008054) entitled preliminary study of selective estrogen modulators (SERMs) combined with maximum androgen blockade for metastatic prostate cancer (see Additional file 1: Table S1). And the study was also registered at the University Hospital Medical Information Network (UMIN ID; 0000064000). All patients provided written informed consent. An analysis was performed and reported to the Internal Review Board in the University of Tokyo Hospital every year.

\section{Study design}

Figure 1 shows the consolidated standards of reporting trials (CONSORT) flow diagram of recruited patients and follow-up. Eligible patients were randomly allocated in a 1:1:1 fashion to receive ADT alone, toremifene plus

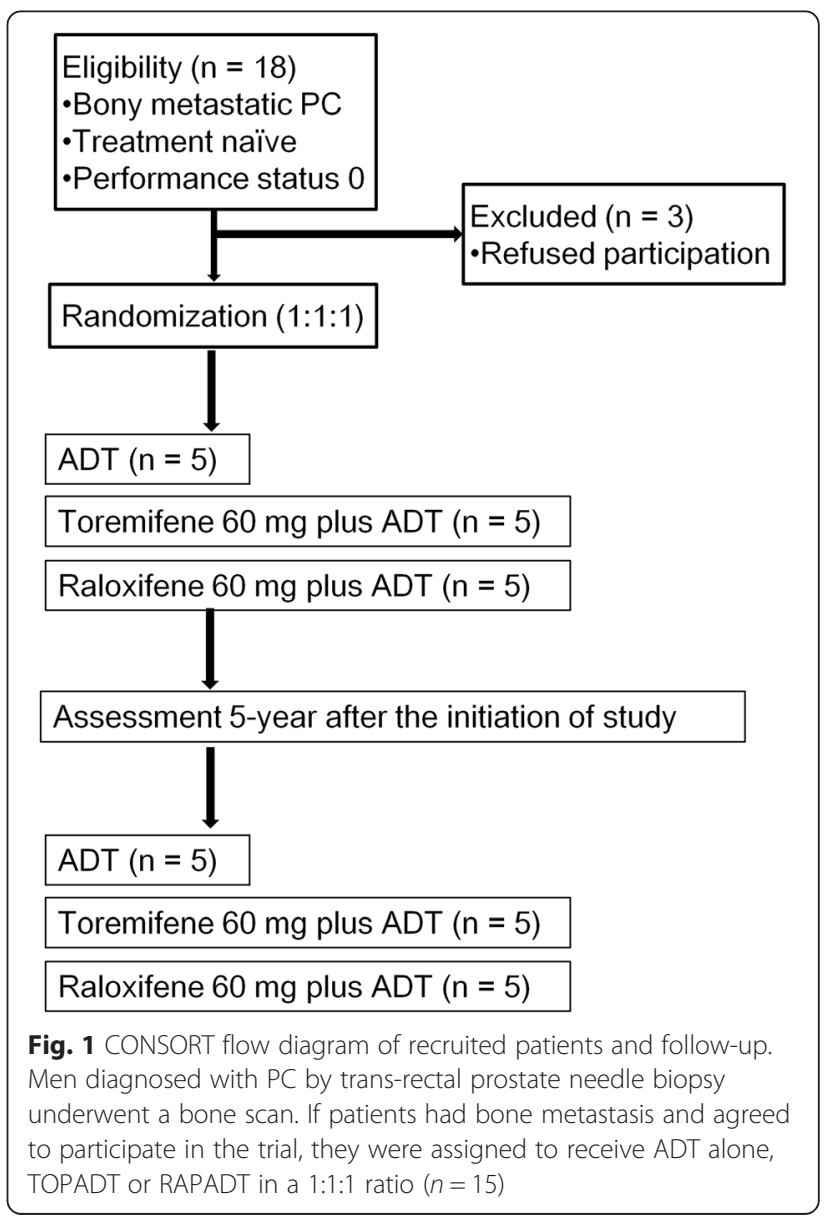


ADT (TOPADT) or raloxifene plus ADT (RAPADT). ADT consisted of castration [bilateral orchiectomy or luteinizing hormone-releasing hormone (LHRH) agonists] combined with $80 \mathrm{mg}$ of bicalutamide. The LHRH agonist was administered throughout, whereas bicalutamide was changed to flutamide on biochemical recurrence after denying anti-androgen withdrawal syndrome. Toremifene (Orion Corporation, Finland) and raloxifene (Eli Lilly Japan K.K.) were given at a dose of $60 \mathrm{mg}$ orally every day combined with aspirin $100 \mathrm{mg}$ daily for prophylactic anti-coagulation.

After PC became hormone-refractory, administration of flutamide was switched to systemic chemotherapy which was performed every 3-4 weeks. If PC became both hormone refractory and chemotherapy-refractory, patients received best supportive care.

\section{Study end points}

Patients were monitored every month during the first year, and every 3 months thereafter. The primary endpoint was the BCR, which was defined as consecutive increase in serum PSA levels to above the patient's PSA nadir [16]. Secondary endpoints included pain on a visual analogue scale (VAS) and functional assessment of cancer therapy (FACT) in every 3 months [17].

\section{Immunohistochemical analysis}

The immunohistochemical analyses for AR, ER $\alpha$ and ER $\beta$ were performed using the streptavidin-biotin amplification method and an EnVision + visualization kit (Dako, Carpinteria, CA, USA) as previously described [9]. The primary antibody against AR (1:40 dilution), ER $\alpha$ (1:40 dilution) and ER $\beta$ (1:200 dilution) was applied and incubated at room temperature for $1 \mathrm{~h}$. The sections were then rinsed in phosphate-bufferes saline and incubated at room temperature with EnVision + for $1 \mathrm{~h}$. The antigenantibody complex was visualized with 3, 3'-diaminobenzidine (DAB) solution [1 mM DAB, $50 \mathrm{mM}$ Tris- $\mathrm{HCl}$ buffer (pH 7.69, and $0.006 \% \mathrm{H}_{2} \mathrm{O}_{2}$ ]. The monoclonal antibodies for AR (AR441) and ERa (NCL-ER-6 F11) were purchased from Dako (Dako, Carpinteria, CA, USA) and Novo-castra Laboratories (Newcastle upon Tyne, UK), respectively. A polyclonal antibody specific for ER $\beta$ was raised in rabbits against peptides synthesized to correspond to the C-terminal region of ER $\beta$ (CSPAEDSKS KEGSQNPQSQ) [9].

\section{Immunohistochemical assessment}

The labeling index (LI) was determined by counting the percentage of cells with positive immunoreactivity per 1000 cells [18]. Two trained pathologists (TF and YY) independently evaluated the tissue sections, and the average LI was used. We defined positive immunoreactivity as showing moderate or strong immunoreactivity.

\section{Statistical analyses}

Correlations between age, pretreatment serum PSA levels, J-CAPRA score [15], and LI were evaluated using the Wilcoxon rank sum test. Associations between the group and clinical parameters including Gleason score (GS) and clinical stage were assessed using chi-square tests. BCR-free survival curves were plotted using the Kaplan-Meier method and verified using the log-rank test and univariate Cox proportional hazards regression models. JMP 11.0.0 software (SAS Institute, Cary, NC, USA) was used for all statistical analyses, and $p<0.05$ was considered to indicate statistical significance.

\section{Results}

\section{Patient characteristics}

From August 14, 2008 to December 27, 2012, 15 patients were enrolled and randomly allocated to either of the three treatment groups as shown in Table 1. The median age was 74 years (range, 63-85). Pretreatment serum PSA levels were 30-8428 ng/mL (median, $198 \mathrm{ng} /$ $\mathrm{mL}$ ). The biopsy samples were evaluated by two pathologists as GS $7(n=3), 8(n=5), 9(n=5)$, or $10(n=2)$. The median J-CAPRA score was 9 (range, 6-11). There was no statistically significant difference in age, serum PSA level, stage, GS, EOD, J-CAPRA score or LI against the anti-AR, -ER $\alpha$, and-ER $\beta$ antibodies among the three groups [ADT v.s. TOPADT and ADT v.s. RAPADT; Fig. 2].

\section{Primary endpoint}

Table 2 shows the PSA response and outcome after ADT with or without SERMs. One patient discontinued toremifene becauseof a headache during the median follow-up period of 1370 days (range, 431-1983). Five (33\%) (2, 2, 1 in the three groups, respectively) patients achieved a PSA-nadir $\leq 0.01$. At the end of the follow-up period, 5 (33\%) patients (3 in the ADT group and 2 in the RAPADT group) experienced $\mathrm{BCR}$ and were switched from bicaltamide to flutamide. One patient in the ADT group became hormone-refractory rapidly and died of PC on day 431 without chemotherapy. One patient in the TOPADT group died of gastric cancer without showing BCR on day 1371 . The BCR-free survival rate was significantly higher in men treated with TOPADT than in those received ADT only $(p=0.04$, ADT vs. TOPADT; $p=0.48$, ADT vs. RAPADT; and $p=$ 0.12 , TOPADT vs. RAPADT; Fig. 3).

Table 3 shows the results obtained from univariate Cox proportional hazards regression models for BCR associated with treatment and the clinicopathological characteristics of the patients, including age, serum PSA levels, J-CAPRA score and LI of AR, ER $\alpha$ and ER $\beta$. TOPADT was only found to be significant in the univariate analysis $\left(p=0.023\right.$; hazard ratio, $\left.1.1 \mathrm{e}^{-9}\right)$. 
Table 1 Baseline characteristics $(n=15)$

\begin{tabular}{|c|c|c|c|c|c|c|}
\hline & & $\operatorname{ADT}(n=5)$ & TOPADT $(n=5)$ & $P$ value (vs. ADT) & RAPADT $(n=5)$ & $P$ value (vs. ADT) \\
\hline Age (median, range) & & $76(74-85)$ & $73(63-81)$ & 0.59 & $72(67-75)$ & 0.29 \\
\hline PSA (ng/mL) (median, range) & & $223(30.6-8428)$ & $264(30-818)$ & 1 & $126(30.8-3600)$ & 0.83 \\
\hline \multirow[t]{4}{*}{ Gleason score } & 7 & 1 & 0 & 0.72 & 2 & 0.57 \\
\hline & 8 & 2 & 2 & & 1 & \\
\hline & 9 & 1 & 2 & & 2 & \\
\hline & 10 & 1 & 1 & & 0 & \\
\hline \multirow[t]{4}{*}{ Clinical T stage } & $2 c$ & 2 & 4 & 0.19 & 2 & 0.55 \\
\hline & $3 a$ & 3 & 1 & & 2 & \\
\hline & $3 b$ & 0 & 0 & & 0 & \\
\hline & 4 & 0 & 0 & & 1 & \\
\hline \multirow[t]{2}{*}{ Clinical N stage } & 0 & 2 & 4 & 0.19 & 1 & 1 \\
\hline & 1 & 3 & 1 & & 4 & \\
\hline \multirow[t]{2}{*}{ Clinical M stage } & $1 b$ & 5 & 4 & 1 & 5 & 1 \\
\hline & 1c & 0 & 1: lung & & 0 & \\
\hline \multirow[t]{3}{*}{ Extent of disease } & 1 & 4 & 3 & 0.49 & 4 & 1 \\
\hline & 2 & 0 & 2 & & 1 & \\
\hline & 3 & 1 & 0 & & 0 & \\
\hline J-CAPRA score (median, range) & & $9(8-10)$ & $8(7-9)$ & 0.37 & $9(6-11)$ & 0.92 \\
\hline \multicolumn{7}{|l|}{ Labeling index (median, range) } \\
\hline$A R$ & & $78.5(54.8-100)$ & $87.1(30-100)$ & 1 & $100(37.5-100)$ & 0.68 \\
\hline ERa & & $27.9(0-46.5)$ & $19.7(0-37.4)$ & 0.34 & $20.6(13.4-35.4)$ & 0.54 \\
\hline ER $\beta$ & & $20.3(2.4-44.9)$ & $11.9(7.4-91.4)$ & 0.75 & $15.2(7-26.4)$ & 0.9 \\
\hline
\end{tabular}

ADT androgen deprivation therapy including surgical or medical castration plus bicalutamide, TOPADT toremifene plus ADT, RAPADT raloxifene plus ADT, J-CAPRA score Japan Cancer of the Prostate Risk Assessment (J-CAPRA) score (ranging from 0 to 12) was calculated on the basis of PSA, Gleason score and clinical stage [15]. Labeling index was determined by counting the percentage of cells with positive immunoreactivity in 1000 cells [18], $A R$ androgen receptor, ER estrogen receptor

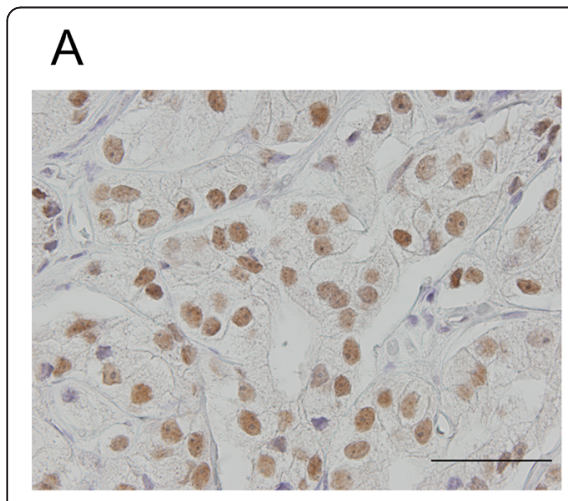

AR

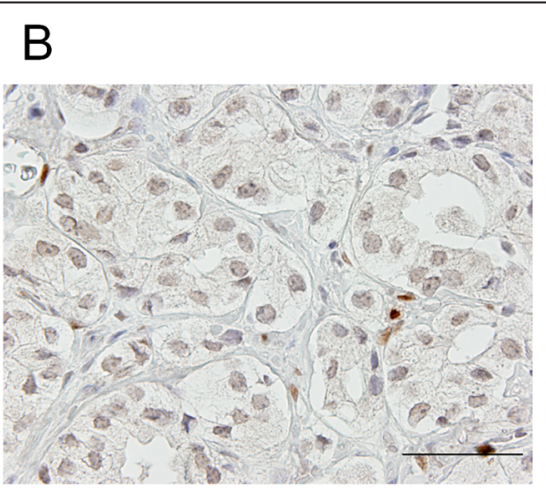

ERa

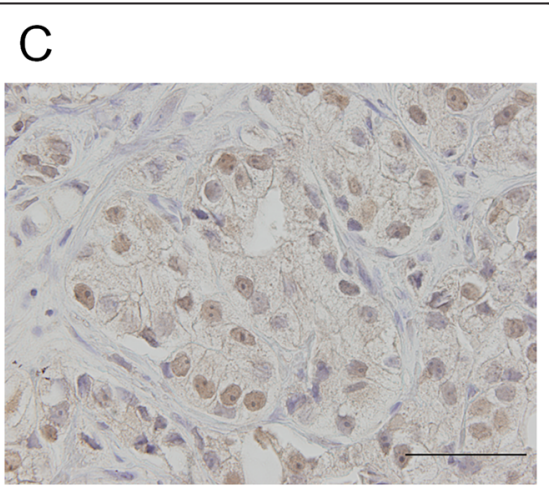

$\mathrm{ER} \beta$

Fig. 2 Immunohistochemical staining for AR (a), ERa (b) and ERß (c) in the tissue sections from the same area of a patient with PC. Strong (a) or moderate (b and $\mathbf{c})$ staining was identified in the nuclei of cancer cells. The LI of AR (a), ERa (b) and ERß (c) in cancer cells was 100, 35.4 and 26.4, respectively. Scale bar $=100 \mu \mathrm{m}$ 
Table 2 PSA response and outcome after ADT with or without selective estrogen receptor modulators $(n=15)$

\begin{tabular}{|c|c|c|c|c|}
\hline & & $\operatorname{ADT}(n=5)$ & TOPADT $(n=5)$ & RAPADT $(n=5)$ \\
\hline \multicolumn{2}{|c|}{ Follow-up period (median, range) } & $1169(431-1631)$ & $1653(730-1983)$ & $1570(750-1883)$ \\
\hline \multirow[t]{3}{*}{ PSA nadir $(n g / m L)$} & $\geq 1$ & 1 & 0 & 1 \\
\hline & $0.01-1.0$ & 2 & 3 & 3 \\
\hline & $\leq 0.01$ & 2 & 2 & 1 \\
\hline \multirow[t]{2}{*}{ Biochemical recurrence } & No & 2 & 5 & 3 \\
\hline & Yes & 3 & 0 & 2 \\
\hline \multirow[t]{3}{*}{ Outcome } & Alive with disease & 4 & 4 & 5 \\
\hline & Died of PC & 1 & 0 & 0 \\
\hline & Died of other disease & 0 & 1: Died of gastric cancer & 0 \\
\hline \multicolumn{2}{|l|}{ Adverse event } & Hot flush: 2 & Hot flush: 3, Headache: 1 & Hot flush: 3 \\
\hline
\end{tabular}

$A D T$ androgen deprivation therapy including surgical or medical castration plus bicalutamide, TOPADT toremifene plus ADT, RAPADT raloxifene plus ADT, $P C$ prostate cancer

Hot flush was observed in all groups; ADT $(n=2)$, TOPADT $(n=3)$, and RAPADT $(n=3)$; although, all patients continued on therapy.

\section{Secondary endpoint}

The VAS scores were significantly decreased after treatment ( $p=0.04$, pre-treatment vs. 3 months thereafter), and showed no statistical differences among the three groups (Fig. 4a). Scores of physical well-being, social well-being, emotional well-being, functional well-being, as well as PC subscale scores of FACT questionnaire, were stable during the follow-up period and not statistically different among the three groups (Fig. 4b-f).

\section{Discussion}

The most common initial therapy for metastatic PC is ADT; however, the durability of ADT is limited and affected by various factors including pretreatment PSA level, GS, tumor stage and PSA nadir [19]. The durability of ADT is also influenced by the ER status of the tumor $[18,20]$.

In fact, estrogens were initially used as one of the earliest forms of treatment agents; however, they were

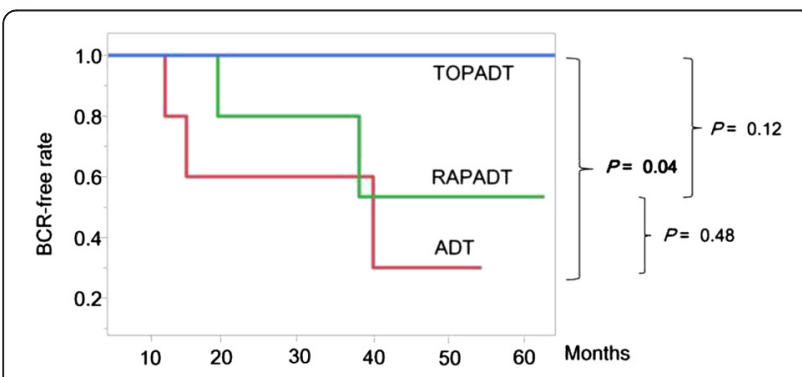

Fig. 3 PSA relapse-free survival rates in men with TOPADT, RAPADT and ADT alone $(n=15)$. The PSA relapse-free survival rate in men treated with TOPADT was significantly greater than in men treated with ADT alone $(p=0.04)$ associated with thromboembolic and cardiovascular side effects [21]. SERMs are synthetic estrogen ligands that can exhibit either estrogenic or anti-estrogenic effects depending on tissue types [22]. Toremifene significantly reduced the incidence of $\mathrm{PC}$ in a transgenic adenocarcinoma mouse prostate model [23], as well as in men with HGPIN [11]. In addition, toremifene increased the bone mineral density of the hip and spine [24] and improved lipid profiles in men receiving ADT for PC [25]. Raloxifene, which acts as an ER agonist in the bone tissue [26], has been developed for the treatment of osteoporosis in women [27] and showed some tumor-inhibitory effects in CRPC in a pilot study [13]. To date, the anticancer effects of these SERMs have not yet been fully investigated in treatment-naïve PC patients. We hypothesized that concurrent use of SERMs would prolong the duration of efficacy of ADT in men with bone metastatic PC.

Currently, we have demonstrated that TOPADT significantly improved the biochemical recurrence rate in men with bone metastatic PC compared with men treated with ADT alone. The results of a recent study

Table 3 Univariate Cox proportional hazard regression models of biochemical recurrence free survival $(n=15)$

\begin{tabular}{lccc}
\hline & \multicolumn{3}{l}{ Univariate } \\
\cline { 2 - 4 } Variable & 3.9 & $0.57-75$ & 0.18 \\
\hline Age $(\geq 74$ vs. $<74)$ & 1.9 & $0.33-15$ & 0.45 \\
Serum PSA $(\mathrm{ng} / \mathrm{mL})(\geq 198$ vs. $<198)$ & 1.7 & $0.27-12$ & 0.57 \\
J-CAPRA $(\geq 9$ vs. $\leq 8)$ & 1.2 & $0.19-8.7$ & 0.87 \\
AR LI $(\geq 93.5$ vs. $<93.5)$ & 0.40 & $0.05-2.5$ & 0.32 \\
ERa LI $(\geq 23.2$ vs. $<23.2)$ & 0.73 & $0.09-4.4$ & 0.73 \\
ERB LI $(\geq 16.1$ vs. $<16.1)$ & $1.1 e^{-9}$ & $0.64-0.64$ & 0.023 \\
TOPADT vs. MAB & 0.5 & $0.069-3.2$ & 0.48 \\
RAPADT vs. MAB & & &
\end{tabular}




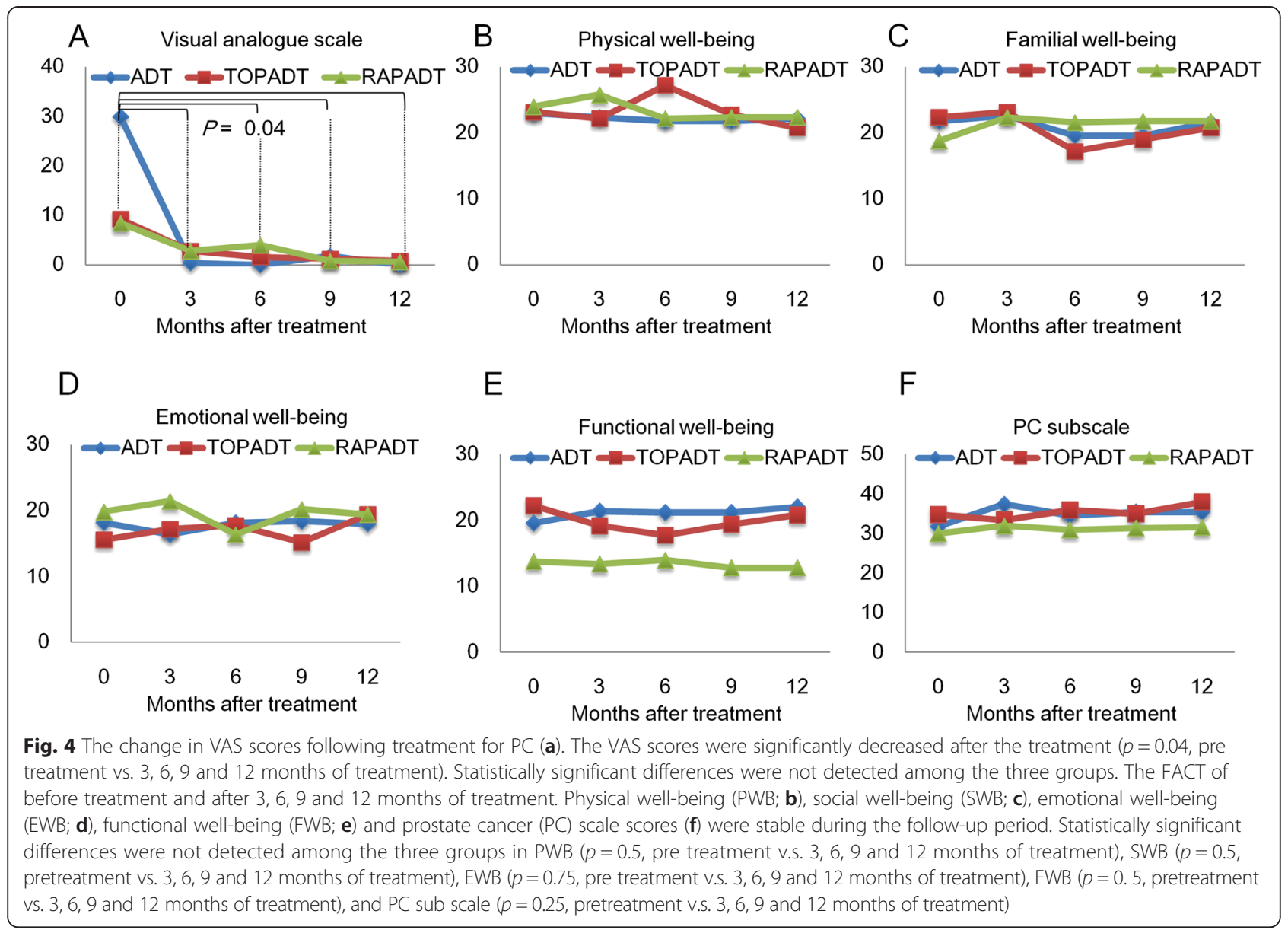

showed that the 5-year BCR-free rate was $30 \%$ in men who received ADT plus docetaxel with median serum PSA levels of 26.7 (range, 5.0-106) [5]. In our study, similar rates were noted for men treated with ADT alone (30 \%). Surprisingly, the 5-year BCR-free rate in the TOPADT group was $100 \%$.

Theoretically, the tumor inhibitory effects of toremifene would be mediated via the suppression of ER $\alpha$ related signals $[28,10]$. ER $\alpha$ expression in PC cells was confirmed by immunohistochemistry and quantitative reverse transcription polymerase chain reaction analyses $[8,9,18]$. The mRNA expression of ER $\alpha$ was much lower than AR in PC cells (1:100 ratio); however, ER $\alpha$ expression in cancer-associated stromal cells was significantly related to cancer-specific survival in men with bone metastatic PC [18]. ER $\alpha$ expression was negatively correlated with survival after radical prostatectomy in locally advanced PC [29]. Additionally, ER $\alpha$ promoted proliferation by regulating $M Y C$ expression and glucose sensitivity in phosphatase and tensin homolog (PTEN)deficient mouse PC cells [8]. Conversely, depletion of $E R \alpha$ inhibited growth in PTEN-deficient mice via a reduction in $M Y C$ protein and alteration of glucose sensitivity [8]. The results of present study demonstrated that toremifene significantly improved the durability of ADT, suggesting blockade of ER $\alpha$ signaling as a potential target for advanced PC.

ER $\beta$ signaling has been associated with a tumorinhibitory effect in PC through both the classical (ER $\beta$ and estrogen-response element complex) and nonclassical pathways (ER $\beta$, Krüppel-like zinc finger transcription factor 5 , and adenosine $3^{\prime}, 5^{\prime}$-monophosphate response element-binding protein-binding protein complex) [30]. ER $\beta$ modulators are expected to inhibit PC growth. Raloxifene exhibits diverse activities via ER depending on whether ER $\alpha$ or ER $\beta$ is expressed in the target organ [26]. The results of the present study did not prove a distinct tumor-inhibitory effect mediated by RAPADT as compared to ADT alone. The difference in the reason tumor inhibitory effect between TOPADT and RAPADT may have been attributed to the potency of the drugs and the pattern of ER expression in PC cells. The tumor-inhibitory effect of fulvestrant, another ER $\beta$ modulator, was limited because the median time to progression was only 4.3 months in men with CRPC treated with fulvestrant [31]. Further investigations of 
additional ER $\beta$ modulators are warranted with respect to their potential role in the inhibition of human PC.

The known adverse events associated with the use of SERMs include hot flushes, sweating, nausea, dizziness, edema, vomiting and thrombosis [27]. In the present study, two men in the ADT group, two men in the TOPADT group, and three men in the RAPADT group complained of mild hot flushes; however, no medical intervention was deemed necessary. Only one man in the TOPADT group discontinued toremifene administration because of a headache. No events of liver dysfunction or thrombosis were observed.

The present study was not without limitations. The sample size was small, and the cohort was limited to a single institution in an all Asian population. A multicenter external validation study would be necessary to further elucidate the additional effect of toremifene on ADT that we found in patients with advanced PC.

\section{Conclusions}

Results from the present study we demonstrated the good clinical efficacy and tolerability of TOPADT in patients with treatment-naïve bone metastatic PC. Additional clinical trials with larger cohorts are warranted to confirm our promising phase IIA results.

\section{Additional file}

Additional file 1: Table S1. CONSORT 2010 checklist of information to include when reporting a cluster randomised trial. (DOCX $30 \mathrm{~kb}$ )

\section{Abbreviations}

ADT: androgen-deprivation therapy; AR: androgen receptor; BCR: biochemical recurrence; CONSORT: consolidated standards of reporting trials; EOD: extent of disease; ER: estrogen receptor; FACT: functional assessment of cancer therapy; J-CAPRA: Japan Cancer of the Prostate Risk Assessment; LI: Labeling index; PC: prostate cancer; PSA: prostate-specific antigen; PTEN: phosphatase and tensin homolog; RAPADT: raloxifene plus ADT; SERM: selective estrogen receptor modulator; TOPADT: toremifene plus ADT; VAS: visual analogue scale.

\section{Competing interests}

The authors declare no conflict of interest.

\section{Authors' contributions}

TF conceived of the study and wrote the draft. ST, SI and $\mathrm{YH}$ revised the draft. HK, YY, MS, HF and TN contributed to data collection and analysis. TU and KT contributed to data interpretation and analysis. All authors approved the final version.

\section{Acknowledgements}

This work was supported by Grants from Yamaguchi Endocrine Research Foundation and by Grants from University of Tokyo Hospital. The sponsors of the study had no role in the study design, data collection, data analysis, data interpretation or writing the draft. Raw data was the property of the University of Tokyo. All authors had final responsibility for the decision to submit for publication.

\section{Author details}

'Department of Urology, Graduate School of Medicine, The University of Tokyo, 7-3-1 Hongo, Bunkyo-ku, Tokyo 113-8655, Japan. '2Department of Urology, Graduate School of Medicine, The Nihon University, Tokyo, Japan.
${ }^{3}$ Department of Geriatric Medicine, Graduate School of Medicine, The University of Tokyo, 7-3-1 Hongo, Bunkyo-ku, Tokyo 113-8655, Japan. ${ }^{4}$ Department of Anti-Aging Medicine, Graduate School of Medicine, The University of Tokyo, 7-3-1 Hongo, Bunkyo-ku, Tokyo 113-8655, Japan.

Received: 16 July 2015 Accepted: 26 October 2015

Published online: 02 November 2015

\section{References}

1. Huggins C, Hodges CV. Studies on prostatic cancer. The effect of castration, of estrogen and of androgen injection on serum phosphatases in metastatic carcinoma of the prostate. Cancer Res. 1941;1:293-7.

2. Cookson MS, Lowrance WT, Murad MH, Kibel AS. American Urological Association. Castration-resistant prostate cancer: AUA guideline amendment. J Urol. 2015;193:491-9.

3. Tannock IF, de Witt R, Berry WR, Horti J, Pluzanska A, Chi KN, et al. Docetaxel plus prednisone or mitoxantrone plus prednisone for advanced prostate cancer. N Engl J Med. 2004;351:1502-12.

4. Petrylak DP, Tangen CM, Hussain MH, Lara PN Jr, Jones JA, Taplin ME, et al. compared with mitoxantrone and prednisone for advanced refractory prostate cancer. N Engl J Med. 2004;351:1513-20.

5. Gravis G, Fizazi K, Joly F, Oudard S, Priou F, Esterni B, et al. Androgendeprivation therapy alone or with or with docetaxel in non-castrate metastatic prostate cancer (GETUG-AFU15): a randomized, open-label, phase 3 trial. Lancet Oncol 2013;14:149-158.

6. Tammela T. Endocrine treatment of prostate cancer. J Steroid Biochem Mol Biol 2004;92:287-295.

7. Culig Z, Steiner H, Bartsch G, Hobisch A. Mechanism of endocrine therapyresponsive and -unresponsive prostate tumors. Endocr Relat Cancer. 2005:12:229-244.

8. Takizawa I, Lawrence MG, Balanathan P, Robello R, Pearson HB, Garg E, et al. Estrogen receptor alpha drives proliferation in PTEN-deficient prostate carcinoma by stimulating survival signaling, MYC expression and altering glucose sensitivity. Oncotarget 2014;6:604-616.

9. Fujimura T, Takahashi S, Urano T, Ogawa S, Ouchi Y, Kitamura T, et al. Differential expression of estrogen receptor beta (ERbeta) and its C-terminal truncated splice variant ERbetacx as prognostic predictors in human prostatic cancer. Biochem Biophys Res Commun 2001;289:692-699.

10. Taneja S, Smith MR, Dalton JT, Raghow S, Barnette G, Steiner M, et al. Toremifene-a promising therapy for the prevention of prostate cancer and complications of androgen deprivation therapy. Expert Opin Investig Drugs 2006;15:293-305.

11. Price D, Stein B, Sieber P, Tutrone R, Bailen J, Goluboff E, et al. Toremifene for the prevention of prostate cancer in men with high grade prostatic intraepithelial neoplasia: results of a double-blind, placebo controlled, phase IIB trial. J Urol 2006;176:965-971.

12. Riggs BL, Hartman LC. Selective estrogen-receptor modulators-mechanisms of action and application to clinical practice. N Engl J Med 2003; 348: 618-629.

13. Shazer RL, Jain A, Galkin AV, Cinman N, Nguyen KN, Natale RB, et al. Raloxifene, an oestrogen-receptor- $\beta$ targeted therapy, inhibits androgenindependent prostate cancer growth: results from preclinical studies and a pilot phase II clinical trial. BJU Int 2006;97:691-697.

14. Soloway MS, Hardeman SW, Hickey D, Raymond J, Todd B, Soloway S, et al. Stratification of patients with metastatic prostate cancer based on disease on initial bone scan. Cancer 1988;61;195-202.

15. Cooperberg MR, Hinotsu S, Namiki M, Ito K, Broering J, Carroll PR, et al. Risk assessment among prostate cancer patients receiving primary androgen deprivation therapy. J Clin Oncol 2009;27:4306-4313.

16. Mottet N, Bellmunt J, Bolla M, Joniau S, Mason M, Matveev V, et al. EAU guidelines on prostate cancer. Part II: treatment of advanced, relapsing, and castration-resistant prostate cancer. Eur Urol. 2011;59:572-583.

17. Hinotsu A, Niimi M, Akaza $\mathrm{H}$, et al. Development of Japanese version of QOL questionnaire for bladder and prostate cancer patients using FACT-BI and P: Pilot study. Gan To Kagaku Ryoho 1999;26:657-666 (In Japanese).

18. Fujimura T, Takahashi S, Urano T, Takayama K, Sugihara T, Obinata D, et al. Expression of androgen and estrogen signaling components and stem cell markers to predict cancer progression and cancer-specific survival in patients with metastatic prostate cancer. Clin Cancer Res 2014;20:4625-4635.

19. Hussain M, Tangen CM, Higano C, Schelhammer PF, Faulkner J, Crawford $E D$, et al. Absolute prostate-specific antigen value after androgen deprivation is a strong independent predictor of survival in new metastatic 
prostate cancer: data from southwest oncology group trial 9346 (INT-0162). J Clin Oncol. 2006;24:3984-90.

20. Kawashima H, Nakatani T. Involvement of estrogen receptors in prostatic tissues. Int J Urol. 2012;19:512-22.

21. Turo R, Smolski M, Esler R, Kujawa ML, Bromage SJ, Oakley N, et al. Diethylstilboestrol for the treatment of prostate cancer: past, present and future. Scand J Urol. 2014;48:4-14.

22. Lonard DM, Smith CL. Molecular perspectives on selective estrogen receptors (SERMs): progress in understanding their tissue-specific agonist and antagonist actions. Steroids. 2002;67:15-24.

23. Raghow S, Hooshdaran MZ, Katiyar S, Steiner MS. Toremifene prevents prostate cancer in transgenic adenocarcinoma of mouse prostate model. Cancer Res. 2002:62:1370-6.

24. Smith MR, Malkowicz SB, Chu F, Forrest J, Price D, Sieber P, et al. Toremifene increase bone mineral density in men receiving androgen deprivation therapy for prostate cancer: Interim analysis of multicenter phase 3 clinical study. J Urol. 2008;179:152-5.

25. Smith MR, Malkowicz SB, Chu F, Forrest J, Sieber P, Barnette KG, et al. Toremifene improves lipid profiles in men receiving androgen deprivation therapy for prostate cancer: Interim analysis of multicenter phase 3 clinical study. J Clin Oncol. 2008;26:1824-9.

26. Dutertre M, Smith CL. Molecular mechanisms of selective estrogen receptors (SERM) action. J Pharmacol Exp Ther. 2000;295:431-7.

27. Draper MW, Flowers DE, Huster WJ, Neild JA, Harper KD, Arnaud C. A controlled trial of raloxifene (LY139481) HCl: impact on bone turnover and serum lipid profile in healthy postmenopausal women. J Bone Miner Res. 1996;11:835-42.

28. Kangas L. Review of the pharmacological properties of toremifene. J Steroid Biochem. 1990;36:191-5.

29. Megas G, Chrisofos M, Anastasiou I, Tsitlidou A, Choreftaki T, Deliveliotis C Estrogen receptor ( $\alpha$ and $\beta$ ) but not androgen receptor expression is correlated with recurrence, progression and survival in post prostatectomy T3NOMO locally advanced prostate cancer. Asian J Androl. 2015;17:98-105.

30. Nakajima Y, Akaogi K, Suzuki T, Osakabe A, Yamaguchi C, Sunahara N, et al. Estrogen regulates tumor growth through a nonclassical pathway that includes the transcription factors ER $\beta$ and KLF5. Sci Signal. 2011;4:1-12.

31. Chadaha MK, Ashraf U, Lawrence D, Tian L, Levine E, Silliman C, et al. Phase II study of fulvestrant (Faslodex) in castration resistant prostate cancer. Prostate. 2008:68:1461-6.

\section{Submit your next manuscript to BioMed Central and take full advantage of:}

- Convenient online submission

- Thorough peer review

- No space constraints or color figure charges

- Immediate publication on acceptance

- Inclusion in PubMed, CAS, Scopus and Google Scholar

- Research which is freely available for redistribution 